\title{
FOCAL DECOMPOSITIONS FOR LINEAR DIFFERENTIAL EQUATIONS OF THE SECOND ORDER
}

\author{
L. BIRBRAIR, M. SOBOLEVSKY, AND P. SOBOLEVSKII
}

Received 10 November 2002

Focal decomposition associated to an ordinary differential equation of the second order is a partition of the set of all two-points boundary value problems according to the number of their solutions. Two equations are called focally equivalent if there exists a homomorphism of the set of two-points problems to itself such that the image of the focal decomposition associated to the first equation is a focal decomposition associated to the second one. In this paper, we present a complete classification for linear second-order equations with respect to this equivalence relation.

\section{Introduction}

The notion of focal decomposition was introduced by Peixoto (see $[2,3,4]$ ) for the topological investigation of differential equations of the second order and for the topological investigation of exponential maps on Riemannian manifolds.

Basically, the focal decomposition of the equation $x^{\prime \prime}=f\left(t, x, x^{\prime}\right)$ can be described in the following way. Consider a 4-dimensional space $\mathbb{R}^{4}$ with coordinates $\left(t_{1}, t_{2}, x_{1}, x_{2}\right)$. A point $\left(t_{1}, t_{2}, x_{1}, x_{2}\right)$ has the index $i$ (note that $i$ can be $\infty$ ) if there are exactly $i$ solutions of the following boundary value problem:

$$
x^{\prime \prime}=f\left(t, x, x^{\prime}\right), \quad x\left(t_{1}\right)=x_{1}, \quad x\left(t_{2}\right)=x_{2} .
$$

Let $\Sigma_{i}(f)$ be the set of points in $\mathbb{R}^{4}$ with the index $i$. The collection $\left\{\Sigma_{i}(f)\right\}$ of these sets is a partition of $\mathbb{R}^{4}$. This partition is called a focal decomposition.

We say that two equations are focally equivalent if there exists a homeomorphism of $\mathbb{R}^{4}$ to itself such that the image of every set $\Sigma_{i}$ for the first equation is the corresponding set $\Sigma_{i}$ for the second one. In other words, a focal equivalence means that the "global behaviour" of the set of all boundary value problems for the first equation is similar to the "global behaviour" of the set of all boundary value problems for the second one. 
Thus, we can consider a question of the focal classification. This question is an analog (but not a direct analog) of the question of topological classification of vector fields or dynamical systems. The difference is the following: in the theory of dynamical systems, one is interested in the global behaviour of all the trajectories, but in our case, we study just the number of solutions.

For some equations, the sets $\Sigma_{i}$ are not necessary manifolds. As was shown by the example of Peixoto and Thom [4], $\Sigma_{i}$ can be subanalytic sets with rather complicated singularities. We analyze here the question of focal classification of linear differential equations-the first and more simple case. As a result, we obtain a complete classification. In particular, we will show that in this case, $\Sigma_{i}$ are $C^{2}$-manifolds.

We consider the following equation: $x^{\prime \prime}=-x$. We define the family of straight lines on the plane $\left\{\left(t_{1}, t_{2}\right)\right\}: t_{2}=t_{1}+\pi k$ (where $k \in Z$ ). For this equation, $\Sigma_{1}=$ $\sigma_{1} \times \mathbb{R}^{2}$, where $\sigma_{1}=\left\{\left(t_{1}, t_{2}\right) \in \mathbb{R}^{2} \mid t_{2} \neq t_{1}+\pi k\right\}$. The sets $\Sigma_{\infty}$ and $\Sigma_{0}$ can be defined as follows:

$$
\begin{gathered}
\Sigma_{\infty}=\left\{\left(t_{1}, t_{2}, x_{1}, x_{2}\right) \in \mathbb{R}^{4} \mid t_{2}=t_{1}+\pi k,\right. \\
\left.\left.x_{1}=x_{2} \text { (if } k \text { is even }\right), x_{1}=-x_{2}(\text { if } k \text { is odd })\right\}, \\
\Sigma_{0}=\mathbb{R}^{4}-\left(\Sigma_{1} \cup \Sigma_{\infty}\right) .
\end{gathered}
$$

Now we give a description of the focal decomposition for all linear differential equations of the second order. Consider a square $(-S, S) \times(-S, S)$ on the plane $\left\{\left(x_{1}, x_{2}\right)\right\}$ with the center $(0,0)$. Note that $S$ can be $\infty$.

Our main result is the following. The focal decomposition of the equation

$$
x^{\prime \prime}=a(t) x^{\prime}+b(t) x+c(t)
$$

can be obtained using a homeomorphism $H:(-S, S)^{2} \times \mathbb{R}^{2} \rightarrow \mathbb{R}^{4}$, preserving fibers, such that

$$
\Sigma_{i}=H\left(\Sigma_{i}(-x) \cap(-S, S)^{2} \times \mathbb{R}^{2}\right)
$$

(see Theorems 3.1 and 3.9.)

Since $H$ preserves fibers, we obtain that $\Sigma_{1}=\sigma_{1} \times \mathbb{R}^{2}$, where $\sigma_{1}$ is the collection of open sets bounded by graphs of some monotone functions. The number of the curves (or the number of the straight lines: $t_{2}=t_{1}+\pi k$ ) intersecting the given square $(-S, S)^{2}$ is the invariant. Observe that this number can be infinite. In this case, the equation $x^{\prime \prime}=a(t) x^{\prime}+b(t) x+c(t)$ is focally equivalent to $x^{\prime \prime}=-x$. It may be interesting to describe the number of the connected components of $\Sigma_{1}$ as a function of coefficients $a(t), b(t)$, and $c(t)$. For example, using the maximum principle, one can show that if $b(t)>0$, then $\Sigma_{1}$ has exactly two connected components, that is, the focal decomposition for the equation is the same as for the equation $x^{\prime \prime}=0$. 
Finally, we state the realization theorem which means that for any picture described above, there exists a second-order differential equation providing this picture.

Note that it could be natural to consider the question from the following viewpoint. A boundary value problem can be reduced to another one with boundary conditions equal to 0 (see [1]). Then one can use the Fredholm theory. But we doubt if this approach can give a geometric structure of the sets $\sum_{i}$. Our approach is based on the consideration of geometric properties of fundamental solutions.

\section{Focal decompositions}

Let

$$
x^{\prime \prime}=f\left(t, x, x^{\prime}\right)
$$

(where $f \in C^{1}\left(\mathbb{R}^{3}, \mathbb{R}\right)$ ) be a second-order differential equation. Let $\Sigma_{i}(f)$ (here $i=0,1, \ldots, \infty)$ be a subset of $\mathbb{R}^{4}$ defined as follows: $\Sigma_{i}(f)=\left\{\left(t_{1}, t_{2}, x_{1}, x_{2}\right) \in \mathbb{R}^{4}\right.$ such that the number of solutions of the boundary value problem $x^{\prime \prime}=f\left(t, x, x^{\prime}\right)$, $x\left(t_{1}\right)=x_{1}, x\left(t_{2}\right)=x_{2}$ is equal to $\left.i\right\}$.

Clearly, $\left\{\Sigma_{i}(f)\right\}$ is a decomposition of $\mathbb{R}^{4}$. This decomposition is called a focal decomposition corresponding to $(2.1)$ (see $[2,3,4])$.

Definition 2.1. Two second-order differential equations $x^{\prime \prime}=f\left(t, x, x^{\prime}\right)$ and $x^{\prime \prime}=$ $g\left(t, x, x^{\prime}\right)$ are called focally equivalent if there exists a homeomorphism $H: \mathbb{R}^{4} \rightarrow$ $\mathbb{R}^{4}$ such that $H\left(\Sigma_{i}(f)\right)=\Sigma_{i}(g)$, for every $i$.

These equations are called strongly focally equivalent if the homeomorphism $H$ can be presented in the following form:

$$
H\left(t_{1}, t_{2}, x_{1}, x_{2}\right)=\left(h\left(t_{1}, t_{2}\right), \tilde{H}_{\left(t_{1}, t_{2}\right)}\left(x_{1}, x_{2}\right)\right),
$$

where $h: \mathbb{R}^{2} \rightarrow \mathbb{R}^{2}$ is a homeomorphism and $\tilde{H}_{\left(t_{1}, t_{2}\right)}: \mathbb{R}^{2} \rightarrow \mathbb{R}^{2}$ is a family of homeomorphisms depending continuously on $\left(t_{1}, t_{2}\right)$.

Remark 2.2. Let $\Sigma_{i}^{T}(f)=\Sigma_{i}(f) \cap\left\{(-T, T) \times(-T, T) \times \mathbb{R}^{2}\right\}$. Hence, $\Sigma_{i}^{T}(f)$ is a decomposition of $(-T, T) \times(-T, T) \times \mathbb{R}^{2}$. We call this decomposition a restriction of the focal decomposition for $f$ to $(-T, T)$.

Definition 2.3. The restriction of the equation $x^{\prime \prime}=f\left(t, x, x^{\prime}\right)$ to $\left(-T_{1}, T_{1}\right)$ is strongly focally equivalent to the restriction of the equation $x^{\prime \prime}=g\left(t, x, x^{\prime}\right)$ to $\left(-T_{2}, T_{2}\right)$ if there exists a homeomorphism $H:\left(-T_{1}, T_{1}\right)^{2} \times \mathbb{R}^{2} \rightarrow\left(-T_{2}, T_{2}\right)^{2} \times$ $\mathbb{R}^{2}$ such that $H\left(\Sigma_{i}^{T_{1}}(f)\right)=\Sigma_{i}^{T_{2}}(g)$ and

$$
H\left(t_{1}, t_{2}, x_{1}, x_{2}\right)=\left(h\left(t_{1}, t_{2}\right), \tilde{H}_{\left(t_{1}, t_{2}\right)}\left(x_{1}, x_{2}\right)\right),
$$


where $h:\left(-T_{1}, T_{1}\right)^{2} \rightarrow\left(-T_{2}, T_{2}\right)^{2}$ is a homeomorphism and $\tilde{H}_{\left(t_{1}, t_{2}\right)}: \mathbb{R}^{2} \rightarrow \mathbb{R}^{2}$ is a family of homeomorphisms depending continuously on $\left(t_{1}, t_{2}\right)$.

Example 2.4. Consider the equation $x^{\prime \prime}=0$. Then

$$
\begin{aligned}
\Sigma_{\infty} & =\left\{\left(t_{1}, t_{2}, x_{1}, x_{2}\right) \mid t_{1}=t_{2}, x_{1}=x_{2}\right\}, \\
\Sigma_{0} & =\left\{\left(t_{1}, t_{2}, x_{1}, x_{2}\right) \mid t_{1}=t_{2}, x_{1} \neq x_{2}\right\}, \\
\Sigma_{1} & =\mathbb{R}^{4}-\left\{\Sigma_{\infty} \cup \Sigma_{0}\right\} .
\end{aligned}
$$

Example 2.5. Consider the equation $x^{\prime \prime}=-x$. Then

$$
\begin{aligned}
\Sigma_{\infty} & =\left\{\left(t_{1}, t_{2}, x_{1}, x_{2}\right) \mid t_{2}=t_{1}+k \pi, x_{1}=(-1)^{k} x_{2}\right\}, \\
\Sigma_{0} & =\left\{\left(t_{1}, t_{2}, x_{1}, x_{2}\right) \mid t_{2}=t_{1}+k \pi, x_{1} \neq(-1)^{k} x_{2}\right\}, \\
\Sigma_{1} & =\mathbb{R}^{4}-\left\{\Sigma_{\infty} \cup \Sigma_{0}\right\} .
\end{aligned}
$$

Remark 2.6. It is easy to see that equation (2.4) is strongly focally equivalent to the restriction of equation (2.5) to $(-\pi / 2, \pi / 2)$. The homeomorphism is given by the following formula: $H\left(t_{1}, t_{2}, x_{1}, x_{2}\right)=\left(h\left(t_{1}\right), h\left(t_{2}\right), x_{1}, x_{2}\right)$, where $h(t)=\arctan t$.

\section{Linear differential equations of the second order}

Theorem 3.1. Consider a linear homogeneous differential equation

$$
x^{\prime \prime}=a(t) x^{\prime}+b(t) x,
$$

where $a(t)$ and $b(t)$ are continuous functions. Then one of the two following statements is true.

(1) Equation (3.1) is strongly focally equivalent to the equation $x^{\prime \prime}=-x$.

(2) There exists an integer number $k \geq 0$ such that (3.1) is strongly focally equivalent to the restriction of the equation $x^{\prime \prime}=-x$ to $(-\pi / 2-\pi k, \pi / 2+\pi k)$.

To prove this theorem, we need some preliminary lemmas. Let $U \subset \mathbb{R}^{4}$ be a plane defined by the equation $x_{1}=x_{2}=0$. Let $\left\{\phi_{1}(t), \phi_{2}(t)\right\}$ be a fundamental solution of (3.1). The pair $\left\{\phi_{1}(t), \phi_{2}(t)\right\}$ can be considered as a map $\phi: \mathbb{R} \rightarrow \mathbb{R}^{2}$ such that $\phi(t)=\left(\phi_{1}(t), \phi_{2}(t)\right)$. Let $\Phi: \mathbb{R} \rightarrow \mathbb{R} P^{1} \simeq S^{1}$ be the standard projectivization of $\phi$ such that $\Phi(t)$ is a 1-dimensional subspace of $\mathbb{R}^{2}$ defined by $\Phi(t)=\left\{z \in \mathbb{R}^{2} \mid z=\lambda \phi(t)\right.$, for some $\left.\lambda \in \mathbb{R}\right\}$. Let $\sigma_{\star} \subset U$ be a subset defined as follows: $\sigma_{\star}=\left\{\left(t_{1}, t_{2}\right) \mid \Phi\left(t_{1}\right)=\Phi\left(t_{2}\right)\right\}$. Let $\sigma_{1}=U-\sigma_{\star}$. Observe that the sets $\sigma_{1}$ and $\sigma_{\star}$ do not depend on a fundamental solution of (3.1) because any fundamental solution can be obtained from $\phi$ using a linear nondegenerate transformation.

Lemma 3.2. The following identity holds: $\Sigma_{1}=\sigma_{1} \times \mathbb{R}^{2}$. 
Proof. By the classical results of the theory of ordinary differential equations, the set $\Sigma_{1}$ is given by inequality

$$
\operatorname{det}\left(\begin{array}{ll}
\phi_{1}\left(t_{1}\right) & \phi_{1}\left(t_{2}\right) \\
\phi_{2}\left(t_{1}\right) & \phi_{2}\left(t_{2}\right)
\end{array}\right) \neq 0
$$

Since inequality (3.2) does not depend on $\left(x_{1}, x_{2}\right)$, we obtain the proposition.

Remark 3.3. Consider equation (2.5) and consider the following fundamental solution: $\phi_{1}(t)=\cos t, \phi_{2}(t)=\sin t$. Then the map $\Phi: \mathbb{R} \rightarrow \mathbb{R} P^{1}$ can be presented in the following form: $\Phi(t)=(\cos 2 t, \sin 2 t)$. Observe that we use the identification of $\mathbb{R} P^{1}$ and $S^{1}$.

Then the set $\sigma_{\star}$ is the collection of the straight lines $t_{2}=t_{1}+k \pi$ (here $k \in \mathbb{Z}$ ) and $\sigma_{1}$ is the collection of open sets between these lines. Observe that the sets $\sigma_{1}$, $\sigma_{\star}$ can be associated with any map $\Phi:\left(T_{1}, T_{2}\right) \rightarrow \mathbb{R} P^{1}$. We are going to use the notations $\sigma_{1}(\Phi), \sigma_{\star}(\Phi)$ for these sets.

Let $\Phi: I_{1} \rightarrow \mathbb{R} P^{1}$ and $\Psi: I_{2} \rightarrow \mathbb{R} P^{1}$ be two continuous maps (here $I_{1}, I_{2} \subset \mathbb{R}$ are open intervals (bounded or unbounded)).

Definition 3.4. The maps $\Phi$ and $\Psi$ are topologically conjugate if there exist a homeomorphism $h: I_{1} \rightarrow I_{2}$ and a homeomorphism $\tilde{h}: \mathbb{R} P^{1} \rightarrow \mathbb{R} P^{1}$ such that the following diagram is commutative:

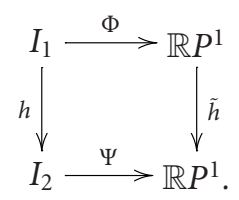

The maps $h$ and $\tilde{h}$ are called conjugations.

Lemma 3.5. Let $\Phi: I_{1} \rightarrow \mathbb{R} P^{1}$ and $\Psi: I_{2} \rightarrow \mathbb{R} P^{1}$ be two topologically conjugate maps. Then there exists a homeomorphism $h_{\star}: I_{1} \times I_{1} \rightarrow I_{2} \times I_{2}$ such that $h_{\star}\left(\sigma_{1}(\Phi)\right)=\sigma_{1}(\Psi)$ and $h_{\star}\left(\sigma_{\star}(\Phi)\right)=\sigma_{\star}(\Psi)$.

Proof. Let $h$ and $\tilde{h}$ be the corresponding conjugations. Define

$$
h_{\star}\left(t_{1}, t_{2}\right)=\left(h\left(t_{1}\right), h\left(t_{2}\right)\right)
$$

Clearly, $h_{\star}$ is a homeomorphism. We prove that $h_{\star}\left(\sigma_{\star}(\Phi)\right)=\sigma_{\star}(\Psi)$. Consider $\left(t_{1}, t_{2}\right) \in \sigma_{\star}(\Phi)$. Then

$$
\Psi\left(h\left(t_{1}\right)\right)=\tilde{h}\left(\Phi\left(t_{1}\right)\right)=\tilde{h}\left(\Phi\left(t_{2}\right)\right)=\Psi\left(h\left(t_{2}\right)\right) .
$$


Thus,

$$
h_{\star}\left(t_{1}, t_{2}\right)=\left(h\left(t_{1}\right), h\left(t_{2}\right)\right) \in \sigma_{\star}(\Psi) .
$$

In the same way, one can prove that $h_{\star}^{-1}\left(\sigma_{\star}(\Psi)\right)=\sigma_{\star}(\Phi)$. The lemma is proved.

Lemma 3.6. Let $\Phi: \mathbb{R} \rightarrow \mathbb{R} P^{1}$ be a projectivization of the fundamental solution $\left(\phi_{1}, \phi_{2}\right)$ of (3.1). Then one of the following propositions is true:

(1) there exists a number $T>0$ such that $\Phi$ is topologically conjugate to the restriction of the map $v$ defined by

$$
v(t)=(\cos 2 t, \sin 2 t)
$$

to the interval $(-T, T)$;

(2) there exists $T>0$ such that $\Phi$ is topologically conjugate to $\left.\nu\right|_{(-T,+\infty)}$;

(3) $\Phi$ is topologically conjugate to $v$ on $\mathbb{R}$.

Proof. Observe that the curve parametrized by $\left(\phi_{1}(t), \phi_{2}(t)\right)$ has no return points because the Wronski determinant is never equal to zero. Let $h_{1}: \mathbb{R} \rightarrow \mathbb{R}$ be a lifting of $\Phi$ to the covering space $\left(\mathbb{R}, v, \mathbb{R} P^{1}\right)$, that is, the following diagram is commutative:

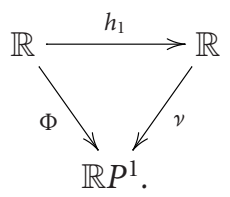

By the observation, $h_{1}$ is a monotone function and, thus, a homeomorphism.

If $\left|\lim _{t \rightarrow \infty} h_{1}(t)\right|=\infty$ and $\left|\lim _{t \rightarrow-\infty} h_{1}(t)\right|=\infty$, then $h=h_{1}$ is a conjugation of $\Phi$ with $\nu$. This corresponds to case (3).

Let $\left|\lim _{t \rightarrow \infty} h_{1}(t)\right|<\infty$ and $\left|\lim _{t \rightarrow-\infty} h_{1}(t)\right|<\infty$. Let $I=\operatorname{Im}\left(h_{1}\right)$. Using some translation in $\mathbb{R}$ and a rotation on $\mathbb{R} P^{1}$, we can transform $I$ into $(-T, T)$ and obtain that $\nu(0)=(1,0)$. It is case $(1)$.

Case (2) is obtained if one of the limits is finite and the other is not.

Now we are going to use the following notation:

$$
S(T)=\left(\left[\frac{2 T}{\pi}\right]+1\right) \frac{\pi}{2}
$$

where $[x]$ is an integer part of $x$.

Lemma 3.7. (1) For each $T>0$, there exists a homeomorphism $H:(-T, T)^{2} \rightarrow$ $(-S(T), S(T))^{2}$ such that

$$
H\left(\sigma_{\star}(\nu) \cap(-T, T)^{2}\right)=\sigma_{\star}(\nu) \cap(-S(T), S(T))^{2} .
$$


(2) Let $S\left(T_{1}\right) \neq S\left(T_{2}\right)$. Then there is no homeomorphism $H:\left(-T_{1}, T_{1}\right)^{2} \rightarrow$ $\left(-T_{2}, T_{2}\right)^{2}$ such that

$$
H\left(\sigma_{\star}(\nu) \cap\left(-T_{1}, T_{1}\right)^{2}\right)=\sigma_{\star}(\nu) \cap\left(-T_{2}, T_{2}\right)^{2} .
$$

(3) For each $T \in \mathbb{R}$, there exists a homeomorphism $H:(T, \infty) \times(T, \infty) \rightarrow \mathbb{R}^{2}$ such that

$$
H\left(\sigma_{\star}(\nu) \cap(T, \infty)^{2}\right)=\sigma_{\star}(\nu)
$$

The lemma is a straightforward consequence of the topological structure of $\sigma_{\star}(\nu)$. Observe that the number $S$ (as well as $T$ ) depends only on (3.1).

Let $V_{t_{1}^{\prime}, t_{2}^{\prime}} \subset \mathbb{R}^{4}=\left\{\left(t_{1}, t_{2}, x_{1}, x_{2}\right)\right\}$ be the plane given by the equations $t_{1}=t_{1}^{\prime}$ and $t_{2}=t_{2}^{\prime}$.

LEMMA 3.8. The set $\Sigma_{\infty}$ has the following structure:

(1) if $\left(t_{1}, t_{2}\right) \in \sigma_{1}$, then $\Sigma_{\infty} \cap V_{t_{1}, t_{2}}=\varnothing$;

(2) if $\left(t_{1}, t_{2}\right) \in \sigma_{\star}$, then $\Sigma_{\infty} \cap V_{t_{1}, t_{2}}$ is a one-dimensional subspace in $V_{t_{1}, t_{2}}$.

Proof. Statement (1) follows from Lemma 3.2. As for statement (2), the set $\Sigma_{\infty}$ $\cap V_{t_{1}, t_{2}}$ can be obtained as the set of solutions of the following system of equations:

$$
\operatorname{det}\left(\begin{array}{ll}
\phi_{1}\left(t_{1}\right) & x_{1} \\
\phi_{1}\left(t_{2}\right) & x_{2}
\end{array}\right)=0, \quad \operatorname{det}\left(\begin{array}{ll}
x_{1} & \phi_{2}\left(t_{1}\right) \\
x_{2} & \phi_{2}\left(t_{2}\right)
\end{array}\right)=0
$$

where $\phi=\left\{\phi_{1}, \phi_{2}\right\}$ is a fundamental solution. Since $\left(t_{1}, t_{2}\right) \in \sigma_{\star}$, the matrix

$$
\left(\begin{array}{ll}
\phi_{1}\left(t_{1}\right) & \phi_{2}\left(t_{1}\right) \\
\phi_{1}\left(t_{2}\right) & \phi_{2}\left(t_{2}\right)
\end{array}\right)
$$

has rank 1. Thus, the set of solutions is a 1 -dimensional subspace in $V_{t_{1}, t_{2}}$.

Observe that the subspace $\Sigma_{\infty} \cap V_{t_{1}, t_{2}}$ is given by an equation of the following type: $x_{1}=\lambda\left(t_{1}, t_{2}\right) x_{2}$, where $\lambda\left(t_{1}, t_{2}\right)=\phi\left(t_{1}\right) / \phi\left(t_{2}\right)$. Note that $\lambda\left(t_{1}, t_{2}\right)$ is well defined, $\lambda\left(t_{1}, t_{2}\right) \neq 0$, for every $\left(t_{1}, t_{2}\right) \in \sigma_{\star}$, and $\lambda\left(t_{1}, t_{2}\right)=1$ if $t_{1}=t_{2}$.

Let $L_{t_{1}, t_{2}}: \mathbb{R}^{2} \rightarrow \mathbb{R}^{2}$ be a linear transformation defined as follows:

$$
L_{t_{1}, t_{2}}\left(x_{1}, x_{2}\right)=\left(x_{1},\left|\lambda\left(t_{1}, t_{2}\right)\right| x_{2}\right) .
$$

Hence, the map $L_{t_{1}, t_{2}}$ transforms the line $x_{1}=\lambda\left(t_{1}, t_{2}\right) x_{2}$ into the line $x_{1}=x_{2}$ if $\lambda\left(t_{1}, t_{2}\right)>0$ and into the line $x_{1}=-x_{2}$ if $\lambda\left(t_{1}, t_{2}\right)<0$. Let $\mu: \mathbb{R}^{2} \rightarrow \mathbb{R}_{+}$be a continuous function such that $\mu\left(t_{1}, t_{2}\right)=\left|\lambda\left(t_{1}, t_{2}\right)\right|$ if $\left(t_{1}, t_{2}\right) \in \sigma_{\star}$. The existence of this function follows from Titze theorem because $\sigma_{\star}$ is a closed subset of $\mathbb{R}^{2}$. 
Let $\tilde{L}: \mathbb{R}^{2} \rightarrow \mathrm{GL}(2, \mathbb{R})$ be a map defined as follows:

$$
\widetilde{L}_{t_{1}, t_{2}}=\left(\begin{array}{cc}
1 & 0 \\
0 & \mu\left(t_{1}, t_{2}\right)
\end{array}\right) .
$$

Hence, if $\left(t_{1}, t_{2}\right) \in \sigma_{\star}$, then $\widetilde{L}_{t_{1}, t_{2}}=L_{t_{1}, t_{2}}$.

Proof of Thereom 3.1. Using Lemmas 3.6 and 3.7, we can construct a homeomorphism $h: \mathbb{R}^{2} \rightarrow(-S, S) \times(-S, S)$ (where $S$ is the number defined in (3.9) or equal to infinity in Lemma 3.6(2) and (3)) such that the image of $\sigma_{1}$ is equal to $\sigma_{1}(\nu) \cap(-S, S)^{2}$ and the image of $\sigma_{\star}$ is equal to $\sigma_{\star}(\nu) \cap(-S, S)^{2}$.

Let $\left(t_{1}, t_{2}\right) \in \sigma_{\star}$. Since any curve $\left(\phi_{1}(t), \phi_{2}(t)\right)$ has no return points, then $\Sigma_{\infty}(-x) \cap V_{h\left(t_{1}, t_{2}\right)}$ is the line $x_{1}=x_{2}$ if $\lambda\left(t_{1}, t_{2}\right)>0$ and it is the line $x_{1}=-x_{2}$ if $\lambda\left(t_{1}, t_{2}\right)<0$. We obtain that the map $H: \mathbb{R}^{4} \rightarrow \mathbb{R}^{4}$, defined by

$$
H\left(t_{1}, t_{2}, x_{1}, x_{2}\right)=\left(h\left(t_{1}, t_{2}\right), \tilde{L}_{t_{1}, t_{2}}\left(x_{1}, x_{2}\right)\right),
$$

is a homeomorphism and we have

$$
\begin{aligned}
H\left(\Sigma_{1}\left(a(t) x^{\prime}+b(t) x\right)\right) & =\Sigma_{1}(-x) \cap(-S, S)^{2} \times \mathbb{R}^{2}, \\
H\left(\Sigma_{\infty}\left(a(t) x^{\prime}+b(t) x\right)\right) & =\Sigma_{\infty}(-x) \cap(-S, S)^{2} \times \mathbb{R}^{2} .
\end{aligned}
$$

Now consider a nonhomogeneous linear differential equation of the second order

$$
x^{\prime \prime}=a(t) x^{\prime}+b(t) x+c(t)
$$

THeorem 3.9. Equation (3.19) is focally equivalent to the corresponding homogeneous equation (3.1). A homeomorphism $H$ can be given in the following form:

$$
H\left(t_{1}, t_{2}, x_{1}, x_{2}\right)=\left(t_{1}, t_{2}, x_{1}+\psi\left(t_{1}\right), x_{2}+\psi\left(t_{2}\right)\right),
$$

where $\psi: \mathbb{R} \rightarrow \mathbb{R}$ is a continuous function.

Proof. It is clear that the sets $\Sigma_{1}$ for both equations are equal. Let $\left(t_{1}, t_{2}\right) \in \sigma_{\star}$. Let $\psi(t)$ be a particular solution of (3.19). The intersection of the sets $\Sigma_{\infty}$ for (3.19) and $V_{t_{1}, t_{2}}$ can be described as the set of solutions of the following equation:

$$
\operatorname{det}\left(\begin{array}{ll}
\phi_{1}\left(t_{1}\right) & x_{1}-\psi\left(t_{1}\right) \\
\phi_{1}\left(t_{2}\right) & x_{2}-\psi\left(t_{2}\right)
\end{array}\right)=0 .
$$

Thus, this set can be obtained from the corresponding set for the homogeneous equation (3.1) using the translation

$$
\left(x_{1}, x_{2}\right) \longrightarrow\left(x_{1}+\psi\left(t_{1}\right), x_{2}+\psi\left(t_{2}\right)\right) \text {. }
$$


Theorem 3.10 (realization theorem). For any $k$, there exists a linear differential equation of the second order focally equivalent to the restriction of the equation of Example 2.5 to $(-T, T)$, where $T=\pi / 2+\pi k$.

This theorem follows from the fact that for any pair of functions $\left(\phi_{1}, \phi_{2}\right)$ with Wronskian different from zero, there exists a linear differential equation of the second order such that $\left(\phi_{1}, \phi_{2}\right)$ is a fundamental solution of this equation.

\section{Acknowledgments}

We are grateful to Mauricio Peixoto and Alberto Verjovsky for useful discussions. The first author was supported by CNPq Grant 300985/93-2 and the third author was supported by FUNCAP.

\section{References}

[1] N. I. Akhieser, Calculus of Variations, Blaisdell Publishing, New York, 1962.

[2] M. M. Peixoto, Focal decomposition in geometry, arithmetic and physics, Geometry, Topology, and Physics (Campinas, 1996) (B. N. Apanasov, S. B. Bradlow, W. A. Rodrigues Jr, and K. K. Uhlenbeck, eds.), de Gruyter, Berlin, 1997, pp. 213-231.

[3] M. M. Peixoto and A. R. da Silva, Focal decomposition and some results of S. Bernstein on the 2-point boundary value problem, J. London Math. Soc. (2) 60 (1999), no. 2, $517-547$.

[4] M. M. Peixoto and R. Thom, Le point de vue énumératif dans les problèmes aux limites pour les équations différentielles ordinaires. I. Quelques exemples, C. R. Acad. Sci. Paris Sér. I Math. 303 (1986), no. 13, 629-633 (French).

L. Birbrair: Departamento de Algebra, Geometria y Topologia, Universidad de Valladolid, 47005 Valladolid, Spain

Current address: Departamento de Matematica, Universidade Federal do Ceará, Fortaleza, Cep. 60155-760, Brazil

E-mail address: lev@agt.uva.es

M. Sobolevsky: Departamento de Matematica, Universidade Estadual do Ceará, Av. Paranjana, 1700, Fortaleza, Cep. 60740-000, Brazil

E-mail address: marina@mat.ufc.br

P. Sobolevskii: Institute of Mathematics, The Hebrew University, Givat Ram, Jerusalem 91904, Israel

E-mail address: pavels@math.huji.ac.il 


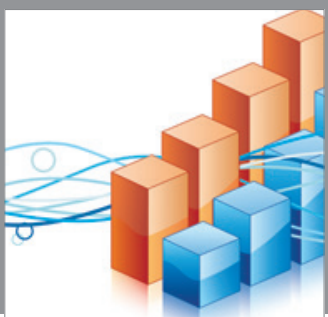

Advances in

Operations Research

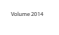

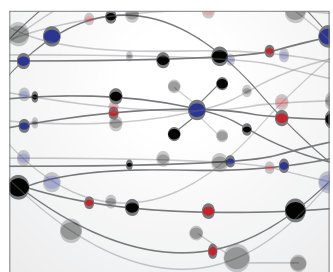

\section{The Scientific} World Journal
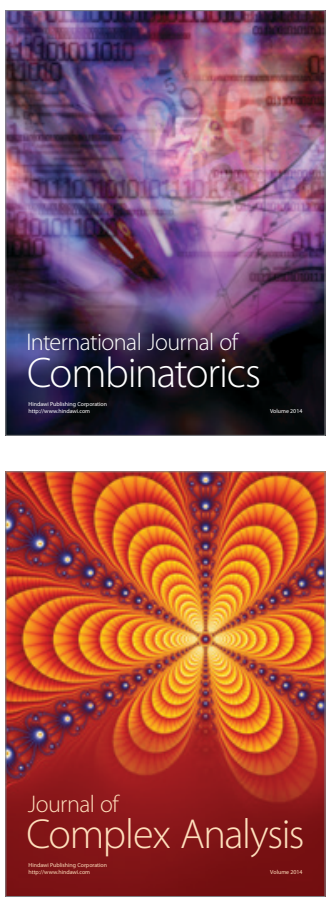

International Journal of

Mathematics and

Mathematical

Sciences
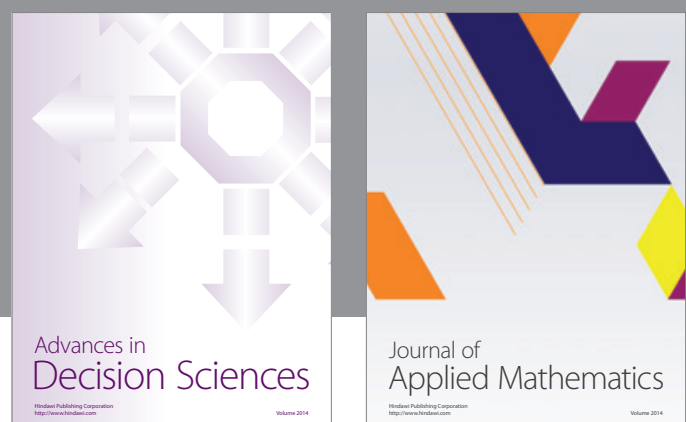

Journal of

Applied Mathematics
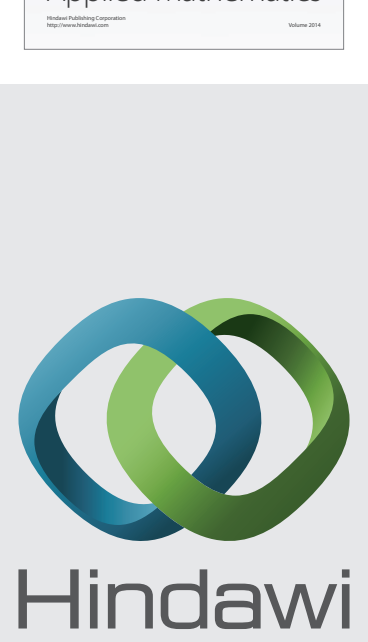

Submit your manuscripts at http://www.hindawi.com
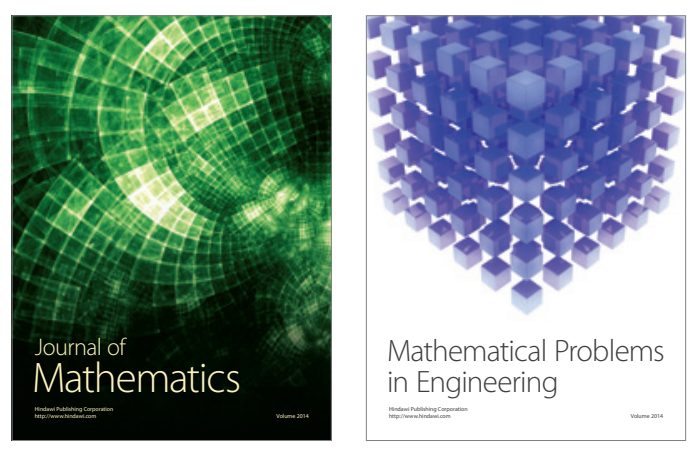

Mathematical Problems in Engineering
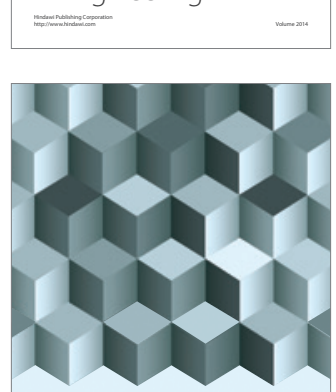

Journal of

Function Spaces
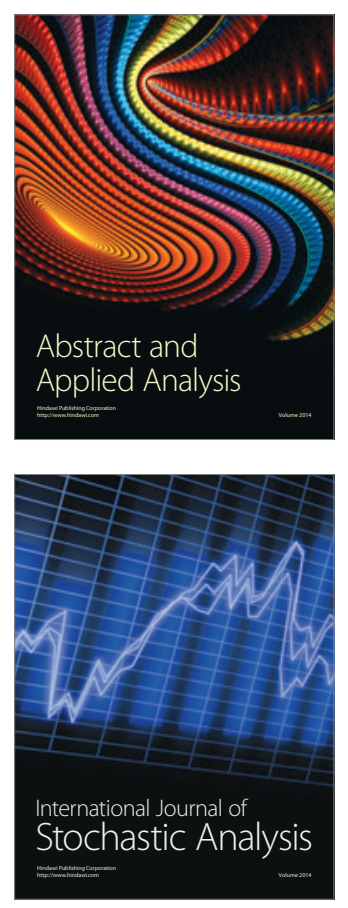

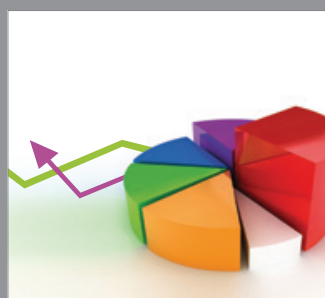

ournal of

Probability and Statistics

Promensencen
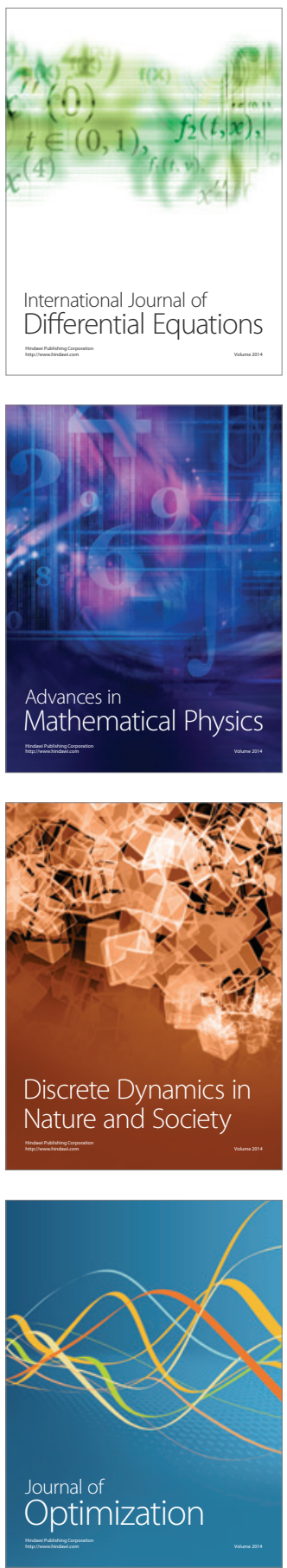